TITLE:

\title{
Vibrational energy relaxation of azulene in the S-2 state. II. Solvent density dependence
}

$\operatorname{AUTHOR}(S)$ :

Yamaguchi, T; Kimura, Y; Hirota, N

\section{CITATION:}

Yamaguchi, T ...[et al]. Vibrational energy relaxation of azulene in the S-2 state. II. Solvent density dependence. JOURNAL OF CHEMICAL PHYSICS 2000, 113(10): 4340-4348

\section{ISSUE DATE:}

2000-09-08

URL:

http://hdl.handle.net/2433/49898

\section{RIGHT:}

Copyright 2000 American Institute of Physics. This article may be downloaded for personal use only. Any other use requires prior permission of the author and the American Institute of Physics. 


\title{
Vibrational energy relaxation of azulene in the $S_{2}$ state. II. Solvent density dependence
}

\author{
T. Yamaguchi, ${ }^{\text {a) }}$ Y. Kimura, ${ }^{\text {b) }}$ and N. Hirota \\ Department of Chemistry, Graduate School of Science, Kyoto University, Kyoto 606-8502, Japan
}

(Received 24 January 2000; accepted 14 June 2000)

\begin{abstract}
We have measured the vibrational energy relaxation of azulene in the $S_{2}$ state in ethane, carbon dioxide, and xenon over a wide density region by the time-resolved fluorescence spectroscopy. The reduced density of the solvent (the density divided by the critical density) has been changed from the gaslike one (lower than 0.05 ) to the liquidlike one ( 2.8 for ethane, 2.7 for carbon dioxide, and 2.0 for xenon). The density dependence of the relaxation rate is compared with that of azulene in the $S_{0}$ state [D. Schwarzer, J. Troe, M. Votsmeier, and M. Zerezke, J. Chem. Phys. 105, 3121 (1996)]. Although the absolute rate largely depends on the electronic state, the density dependence of the relaxation rates is roughly the same for both electronic states, which can be considered as a side-proof of the isolated binary collision model. Possible origins for the validity of the isolated binary collision model are discussed. (C) 2000 American Institute of Physics.
\end{abstract}

[S0021-9606(00)51534-7]

\section{INTRODUCTION}

The isolated binary collision (IBC) model is one of the simplest theories for the vibrational energy relaxation in liquids. In this model, the relaxation rate $\left(k_{c}\right)$ is described by the following equation:

$$
k_{c}=Z P \text {, }
$$

where $Z$ is the collision frequency between the solute and the solvent molecules, and $P$ is the averaged relaxation probability per collision, whose value is the same as that in the gas phase reaction. The validity of the IBC model has been a topic in the solution chemistry during the latter half of this century, but it is yet to be resolved.

In the theory on the ultrasonic propagation in liquids, Kittel implicitly assumed the IBC model for the vibrational energy relaxation for the first time. ${ }^{1}$ Later, Litovitz et al. explicitly proposed the IBC model for the vibrational energy relaxation in liquids, and explained their experimental data on neat carbon disulfide and carbon dioxide. ${ }^{2}$ In their work, they invented the moving wall cell model to estimate the collision frequency in a dense fluid, which agrees well with their experimental data. However, they showed at the same time that the value of the collision frequency depends very much on the way of estimation. In response to the papers of Litovitz et al., there were many theoretical discussions on the validity of the IBC model. One of the strongest criticisms against the IBC model was that the many body interference effect should be included when the collision frequency becomes as high as the frequency of the intramolecular vibration. ${ }^{3,4}$ The arguments on the vibrational energy relaxation in the earlier days are summarized in reviews by Oxtoby. ${ }^{5}$ Meanwhile, experimental and theoretical studies

\footnotetext{
${ }^{a)}$ Present address: Institute for Chemical Research, Kyoto University, Gokasho, Uji, Kyoto 611-0011, Japan.

${ }^{b)}$ Electronic mail: ykimura@kuchem.kyoto-u.ac.jp
}

were continued on the compressed diatomic or triatomic gases in order to test the validity of the IBC model. ${ }^{6-12}$

The situation changed in the last decade due to the development of the short-pulsed laser and its application to so-called supercritical fluids. Using the fluid above the critical temperature $\left(T_{c}\right)$, we can change the density of fluids from the gaslike one to the liquidlike one continuously without any phase transition. This particular property of supercritical fluids makes them suitable solvents to examine the IBC model, because we can gradually increase the density of the solvent from the gaslike one where the IBC model is valid. In addition, the short pulse laser technology made it possible to measure the fast (order of ps) vibrational energy relaxation of dilute solute molecules. There have been several works performed on the dilute solute molecules in supercritical fluids during the past decade. ${ }^{13-22}$ Paige and Harris studied the vibrational energy relaxation of molecular iodine $\left(\mathrm{I}_{2}\right)$ in the ground electronic state in xenon $(\mathrm{Xe})$ near the liquidlike densities. ${ }^{13}$ They found that the vibrational energy decay curves at different densities coincide with each other by scaling the time axis, which indicates that the vibrational energy relaxation dynamics of $\mathrm{I}_{2}$ is characterized only by a single parameter. They also performed molecular dynamics simulations to estimate the collision frequency. By adjusting the contact distance, they could reproduce the relative relaxation rates by the IBC model. Harris et al. also measured the vibrational energy relaxation of the vibrationally high excited azulene in the $S_{0}$ state in supercritical fluids including Xe. ${ }^{14}$ From the difference in the density and temperature dependence between azulene and iodine, they concluded that the rigid cluster is formed in the case of azulene in Xe.

Schwarzer et al. measured the vibrational cooling of the vibrationally highly excited azulene in the $S_{0}$ state in carbon dioxide $\left(\mathrm{CO}_{2}\right)$, ethane $\left(\mathrm{C}_{2} \mathrm{H}_{6}\right)$, and $\mathrm{Xe}$ over a wide density region from the gaslike to the liquidlike ones. ${ }^{15}$ They also 
performed the Monte Carlo simulation assuming the attractive hard sphere potential, and estimated the collision frequency from the density at the contact distance. ${ }^{16}$ They succeeded in reproducing the density dependence of the relaxation rates in $\mathrm{C}_{2} \mathrm{H}_{6}, \mathrm{Xe}$, and $\mathrm{C}_{2} \mathrm{H}_{6}-\mathrm{Xe}$ mixtures by the results of the simulation, and proposed that the IBC model can explain the vibrational energy relaxation in fluids. However, the attractive potential they used was much larger than that expected from the Lennard-Jones parameters and the Lorentz-Berthelot rule. ${ }^{16 \mathrm{~b}}$ After that, Heidelbach et al. performed the molecular dynamics simulation including the internal degree of freedom of the solute. ${ }^{17}$ They reported that the energy transfer occurs exclusively from the low frequency modes in the low-density region, whereas the high frequency modes can contribute to the energy transfer to some degree in the high-density region.

Urdahl et al. studied the vibrational energy relaxation of the well-separated mode of the polyatomic molecule [asymmetric $\mathrm{C}=\mathrm{O}$ stretching mode of tungsten hexacarbonyl $\left(\mathrm{W}(\mathrm{CO})_{6}\right)$ ] by the transient infrared (IR) spectroscopy. ${ }^{19}$ They measured the density and temperature dependence around the critical points of the solvents in detail. They found that the temperature dependence is inverted around the critical point. They devised a hydrodynamic theory for the vibrational energy relaxation in supercritical fluids, and proposed that the particular behavior around the critical point is related to the long-range density fluctuation that is the reason for the critical anomaly. ${ }^{20}$ In response to their proposal, Goodyear and Tucker performed a molecular dynamics simulation, and showed that the small density dependence of the relaxation rates around the critical point can be explained by the local density enhancement of the solvents around the solute. ${ }^{21}$ We recently showed that there are some internal inconsistencies in the hydrodynamic theory of Cherayil and Fayer. ${ }^{23}$

In addition to the experimental studies above, computational studies have also been developed. For example, Chesnoy and Weis found in their molecular dynamics (MD) simulation that the short-time behavior of the force-force time correlation function is almost reproduced by its self-part. ${ }^{7}$ Adelman et al. showed in their theoretical calculation that the density dependence of the vibrational energy relaxation rate is dominated by that of the initial value of the forceforce time correlation function. ${ }^{9}$ Recently, Goodyear and Stratt showed that the functional form of the high frequency part of the friction spectrum is almost independent of the solvent density, ${ }^{10}$ and Larsen and Stratt found that the high frequency part of the friction spectrum is reproduced by the relative motion between a solute and a nearest-neighbor solvent. ${ }^{12}$ All these theoretical models for diatomic solute cases suggests that the IBC model holds over the wide solvent density region to some extent.

Looking over the history around the experimental examination of the IBC model for the vibrational energy relaxation in fluids, it is noticed that the estimation of the collision frequency $(Z)$ has been essential for the examination of the IBC model since the study of Litovitz. ${ }^{2}$ Although the rapid development of the computer technology made the molecular simulation available for ordinary experimental chemists, the

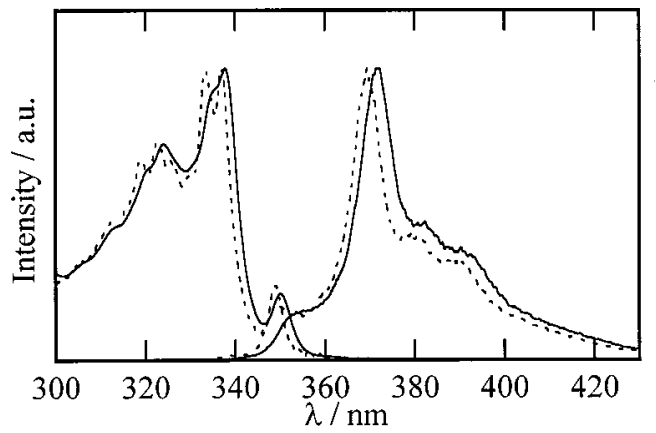

FIG. 1. The absorption (left) and the fluorescence (right) spectra of azulene in the $S_{2}$ state in $\mathrm{CO}_{2}$. Dotted curves: $\rho_{r}=0.2$, solid curves: $\rho_{r}=1.8$. The temperature is $323 \mathrm{~K}$ at both densities. The $0-0$ band of the fluorescence at $\rho_{r}=1.8$ suffers from the reabsorption effect.

results of the simulation strongly depends on the intermolecular potential or the other parameters, ${ }^{13,16}$ especially in the medium-density region where the attractive interaction is the dominant factor to determine the equilibrium structure. Considering the progress of the experimental techniques to measure the vibrational energy relaxation rates, the estimate of the collision frequency appears to be a bottleneck in the examination of the IBC model.

In this work, we try to test the IBC model for the vibrational energy relaxation in fluids experimentally, trying to avoid the arbitrariness of the estimation of the collision frequency. What we have actually done is to measure the vibrational energy relaxation rates of azulene in the $S_{2}$ state in fluids such as $\mathrm{CO}_{2}, \mathrm{C}_{2} \mathrm{H}_{6}$, and $\mathrm{Xe}$, and to compare them with those in the $S_{0}$ state. Azulene emits a relatively strong emission from the $S_{2}$ state, whose lifetime is about 2 ns. The solvent effects on the absorption and the fluorescence spectra of the $S_{0}-S_{2}$ transition of azulene is not so large, as is shown in Fig. 1. The fluorescence peak shift is no larger than +5 $\mathrm{nm}\left(-400 \mathrm{~cm}^{-1}\right)$ in the density region of all solvent fluids in this experiment. It means that the electronic transition does not cause a large change of the solute-solute interaction. Therefore, we expect that the collision frequency does not depend largely on the electronic states, and that the density dependence of the relaxation rates does not either, if the IBC model holds in both electronic states. Since the fluorescence band shape strongly depends on the intramolecular vibrational excess energy, we can measure the vibrational energy relaxation in the $S_{2}$ state in solution from the time-resolved fluorescence spectra. The details of the experimental methods and the results in gases and in liquids are presented in Ref. 24 (hereafter called paper I). As is introduced above, Schwarzer et al. determined the solvent density dependence of the relaxation rates in the $S_{0}$ state in supercritical fluids from the gaslike to the liquidlike densities. ${ }^{15}$ Our results on $S_{2}$ azulene are compared with their results on $S_{0}$ azulene in this paper.

\section{EXPERIMENT}

The experimental procedures to measure the timeresolved fluorescence spectra by a streak camera are described elsewhere. ${ }^{24-26}$ Briefly, the second harmonic of the output of a mode-locked dye laser (Coherent 700, $569 \mathrm{~nm}$ ) 
was used as the excitation light. The saturable absorber was used in the experiment in $\mathrm{CO}_{2}$ and $\mathrm{C}_{2} \mathrm{H}_{6}$, and the pulse width was about 3 ps, whereas it was not used in the experiment in Xe (pulse width $\sim 7 \mathrm{ps}$ ). The polarization of the excitation light was set to the magic angle. The fluorescence was corrected at the right angle, and focused onto the slit of a spectrograph (Chromex, 250IS) after the depolarization. The time-resolved fluorescence was detected by a streak camera (Hamamatsu, C4334). The full width at half-maximum (FWHM) of the response function was about 30 ps.

The high-pressure apparatus for the measurement of fluorescence in supercritical fluids is described elsewhere. ${ }^{24-28}$ We used two different high pressure cells for the pressure higher or lower than $35 \mathrm{MPa}$. The cell for the lower pressure is made of titanium and has four quartz windows of larger aperture. ${ }^{29}$ The cell for the higher pressure is made of titanium and has three quartz windows of relatively small aperture. The temperature of the cell was controlled by flowing the thermostated water or silicon oil through the cell. We made measurements mainly at two different temperatures, $323 \mathrm{~K}$ and $385 \mathrm{~K}$. The latter is the temperature where the vibrational energy relaxation rates in the ground state have been reported. ${ }^{15}$ In some measurement at higher pressure near the limit of the high-pressure cells, the temperature was decreased in order to reduce the pressure at the same density. The pressure was measured by strain gauges $(\mathrm{Ky}-$ owa PGM $500 \mathrm{KH}$ below $35 \mathrm{MPa}$ and Nagano Keiki KH78161 above $35 \mathrm{MPa}$ ), and the density was calculated from the empirical equations of states. ${ }^{30}$ A hand-operated hydraulic pressure generator was used to achieve the pressure higher than $35 \mathrm{MPa}$. We could compress the fluids up to liquidlike densities $\left[\rho_{r}=2.8\right.$ for $\mathrm{C}_{2} \mathrm{H}_{6}$ and $\rho_{r}=2.7$ for $\mathrm{CO}_{2}$, where $\rho_{r}$ stands for the reduced density (the density divided by the critical density)] by applying the pressure of about $200 \mathrm{MPa}$ at $323 \mathrm{~K}$. We used $\mathrm{CO}_{2}$ (Sumitomo Seika, >99.99\%), $\mathrm{C}_{2} \mathrm{H}_{6}$ (Sumitomo Seika, $>99.0 \%$ ), and Xe (Iwatani, $>99.995 \%$ ) as solvent fluids without purification. Azulene (Nacalai Tesque) was purified twice by sublimation before use. The concentration of the sample was less than $10^{-1} \mathrm{mM}$. The absorption spectra were measured by an UV spectrometer (Shimadzu, UV2500PC).

\section{RESULTS}

The time-resolved fluorescence spectra in fluids at $323 \mathrm{~K}$ are shown in Fig. 2. The narrowing and the blueshift of the fluorescence spectra are observed in all fluids at every density, although the time scale of the change of the fluorescence band shape depends on both the solvent densities and the solvent species. This change is due to the loss of the intramolecular vibrational excess energy, and we can determine the transient intramolecular vibrational excess energy by fitting the transient spectra to the stationary vibrationally hot fluorescence spectra of the isolated molecule in the vapor phase. The details of the fitting procedure are described in paper I. ${ }^{24}$ In the fitting procedure, it was required to shift the spectra of the isolated molecule in order to calibrate the spectral shift due to the solvent. The amount of the shift is at most $+5 \mathrm{~nm}\left(\sim-400 \mathrm{~cm}^{-1}\right)$ at the highest density of the $\mathrm{C}_{2} \mathrm{H}_{6}$ in this experiment. Solely shifting the spectra could
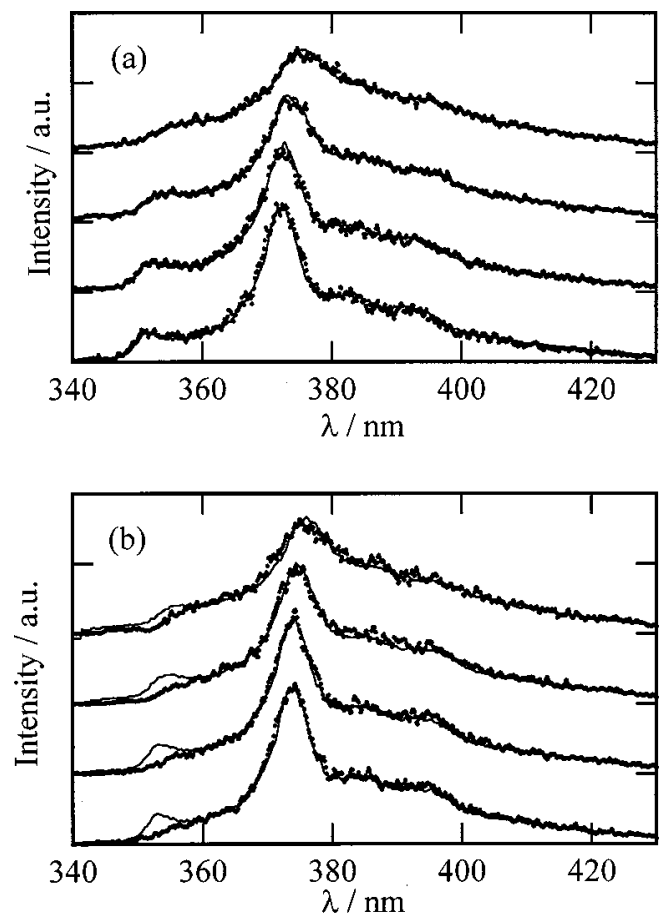

FIG. 2. Time dependence of the fluorescence spectrum in fluids. (a) In Xe at $\rho_{r}=1.0$. From upper to lower, at 49 ps (estimated excess energy: 3420 $\left.\mathrm{cm}^{-1}\right), 101 \mathrm{ps}\left(1680 \mathrm{~cm}^{-1}\right), 199 \mathrm{ps}\left(480 \mathrm{~cm}^{-1}\right)$, and $500 \mathrm{ps}\left(130 \mathrm{~cm}^{-1}\right) .(\mathrm{b})$ In $\mathrm{C}_{2} \mathrm{H}_{6}$ at $\rho_{r}=2.6$. From upper to lower, at $1.6 \mathrm{ps}\left(2500 \mathrm{~cm}^{-1}\right), 19.7 \mathrm{ps}$ $\left(970 \mathrm{~cm}^{-1}\right), 40.4 \mathrm{ps}\left(230 \mathrm{~cm}^{-1}\right)$, and $100 \mathrm{ps}\left(60 \mathrm{~cm}^{-1}\right)$. Filled circle: experiment, solid curve: fitting. The discrepancy around the $0-0$ band (b) is due to the reabsorption effect.

reproduce the spectra in $\mathrm{C}_{2} \mathrm{H}_{6}$ and Xe. In particular, we did not observe any extra broadening around the critical density in any fluids. However, we had to consider the solvent induced broadening in the case of $\mathrm{CO}_{2}$, probably due to the multipolar character of $\mathrm{CO}_{2} \cdot{ }^{31}$ The solvent reorganization energy in $\mathrm{CO}_{2}$ estimated as is done in paper $\mathrm{I}$ is at most 40 $\mathrm{cm}^{-1}$ at the highest density. We consider the value so small that the solvation dynamics affect little the temporal evolution of the fluorescence band shape. The results of the fittings are shown in Fig. 2 by solid curves, which reproduce well the experimental transient spectra. The discrepancies around the $0-0$ band in Fig. 2(b) is due to the reabsorption effect.

The time evolutions of the intramolecular vibrational energy determined by the above procedure are shown in Fig. 3 together with the time profiles of the total fluorescence intensity. The vibrational energy relaxation rates were determined by fitting the time profiles of both the vibrational excess energies and the total fluorescence intensities simultaneously. The initial intramolecular vibrational energy was fixed to $6430 \mathrm{~cm}^{-1}{ }^{24}$ The simulated time profiles are shown in Fig. 3 by solid curves. The instrumental response function is also shown in Fig. 3(b) as a dotted curve. The fitting procedure works well in all fluids at every density. A small dip of the total fluorescence intensity around $450 \mathrm{ps}$ in Fig. 3(a) is due to the damage of the MCP plate of our streak camera. The errors of the relaxation rates are estimated to be about $10 \%$.

In order to analyze the fluorescence spectra at the el- 

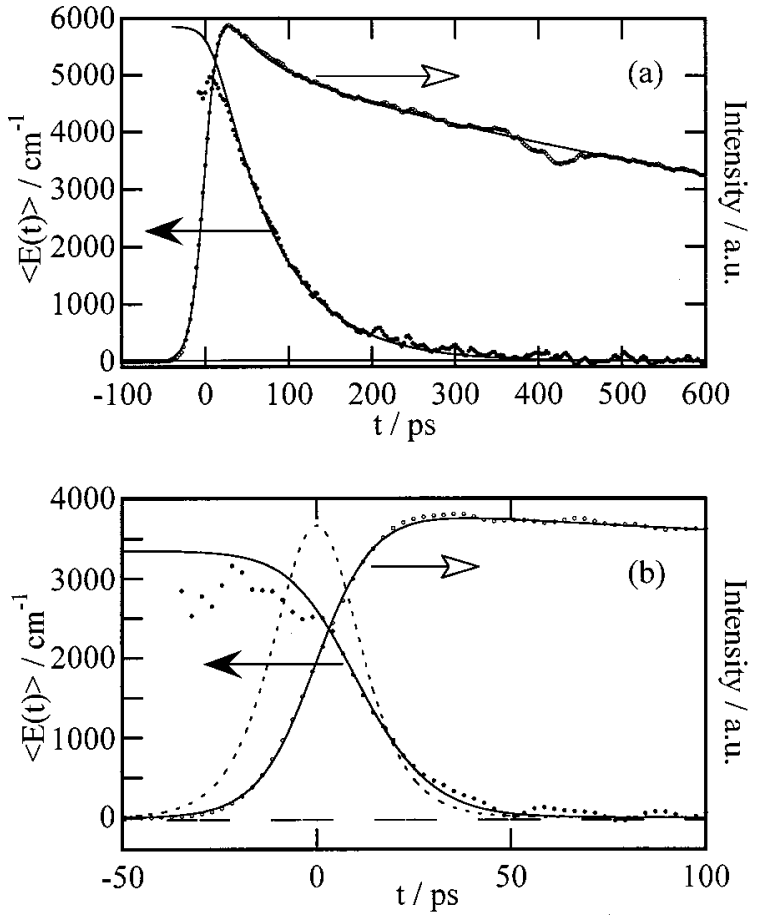

FIG. 3. Time dependence of the intramolecular vibrational excess energy in fluids. (a) In Xe at $\rho_{r}=1.0$. (b) In $\mathrm{C}_{2} \mathrm{H}_{6}$ at $\rho_{r}=2.6$. Filled circle: $\langle E(t)\rangle$; open circle: fluorescence intensity; solid curve: simulation; dotted curve: response function.

evated temperature $(385 \mathrm{~K})$, we had to modify the fitting procedure, since the relaxed fluorescence band shape depended on the temperature. In Fig. 4, we compare the relaxed fluorescence in $\mathrm{CO}_{2}$ at $385 \mathrm{~K}$ and $\rho_{r}=0.05$ with that in argon at $341 \mathrm{~K}$ and 20 bar. The relaxed fluorescence band shapes are the same in $\mathrm{C}_{2} \mathrm{H}_{6}, \mathrm{Xe}$, and $\mathrm{CO}_{2}$ in the low-density region if we compare at the same temperature. Since the band shapes of the relaxed fluorescence in dilute gases do not depend on the solvent species, the difference in Fig. 4 can be considered as the temperature effect. It is quite natural that the band shape of the relaxed fluorescence changes with the temperature, since the intramolecular vibrational energy at thermal equilibrium depends on the temperature. We simulated the relaxed fluorescence at $385 \mathrm{~K}$ by the vibrational

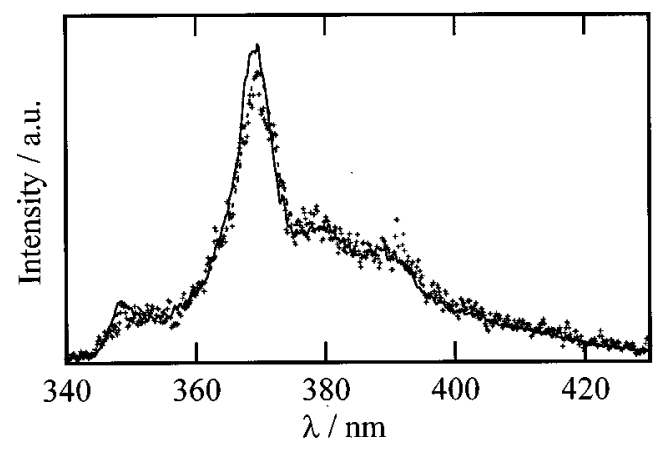

FIG. 4. Relaxed fluorescence spectrum in $\mathrm{CO}_{2}$ at $\rho_{r}=0.05$ and $385 \mathrm{~K} .+$ : Experimental spectrum at $1500 \mathrm{ps}$. The solid curve is the relaxed fluorescence at $341 \mathrm{~K}$ and the dotted one is the fluorescence with the $600 \mathrm{~cm}^{-1}$ of the excess energy.

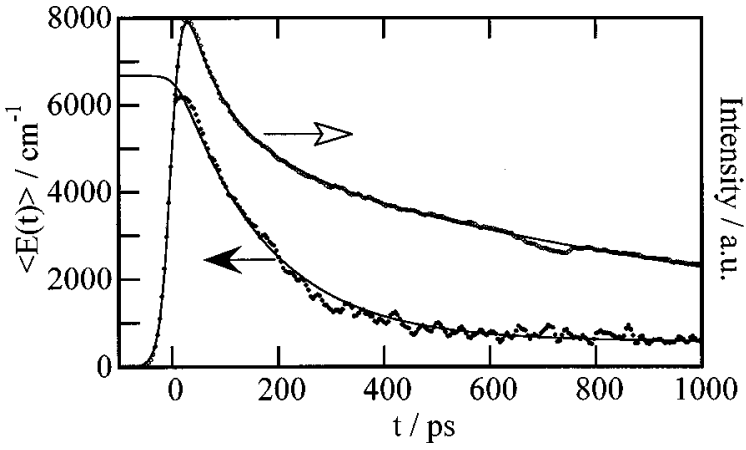

FIG. 5. Time dependence of the vibrational excess energy in $\mathrm{CO}_{2}$ at $\rho_{r}$ $=0.05$ and $385 \mathrm{~K}$. Filled circle: $\langle E(t)\rangle$; open circle: fluorescence intensity; solid curve: simulation.

excess energy dependence of the fluorescence band shapes at $341 \mathrm{~K}$ to find that the vibrational excess energy of $\sim 600$ $\mathrm{cm}^{-1}$ (dashed curve in Fig. 5) can reproduce the relaxed fluorescence spectrum at $385 \mathrm{~K} .{ }^{32}$

The time profile of the intramolecular vibrational excess energy in $\mathrm{CO}_{2}$ at $385 \mathrm{~K}$ and $\rho_{r}=0.05$ are shown in Fig. 5. The value of $\langle E(t)\rangle$ (the intramolecular vibrational excess energy) does not relax to zero due to the temperature dependence of the relaxed fluorescence spectrum. We need to subtract the increase of the intramolecular thermal energy from the value of $\langle E(t)\rangle$ at $385 \mathrm{~K}$. In order to take its effect into account, we altered Eq. (7) in paper I as follows,

$$
I_{F}(t)\langle E(t)\rangle=\int_{-\infty}^{t} d t^{\prime} g\left(t^{\prime}\right) f\left(t-t^{\prime}\right)\left[e\left(t-t^{\prime}\right)+E_{b}\right],
$$

where the notations are the same as in paper I, and $E_{b}$ is the baseline of $\langle E(t)\rangle$, which corresponds to the increase of the intramolecular vibrational thermal energy from that at $341 \mathrm{~K}$. We analyzed the experimental time profiles by Eq. (2), which is shown in Fig. 5 as solid curves. We analyzed the time-resolved fluorescence at $385 \mathrm{~K}$ and in the higherdensity region in the same procedure. The shift and the broadening factor were determined so that the value of $E_{b}$ does not depend on the solvent density. The broadening factor is not required in the case of $\mathrm{C}_{2} \mathrm{H}_{6}$ and $\mathrm{Xe}$.

The results of the vibrational energy relaxation rates are summarized in Figs. 6-8. The relaxation rates in the $S_{2}$ state are the filled marks, and those in the $S_{0}$ state are the open circles. The longitudinal axes are scaled so that the marks for both states exist approximately at the same position in the high-density region. The scaling factors are 1.4, 2.1, and 1.9 for $\mathrm{C}_{2} \mathrm{H}_{6}, \mathrm{CO}_{2}$, and $\mathrm{Xe}$, respectively. The density dependence of the relaxation rates in both states agrees with each other approximately. In particular, the agreement in the highdensity region $\left(\rho_{r}>2.0\right)$ of $\mathrm{C}_{2} \mathrm{H}_{6}$ and $\mathrm{CO}_{2}$ is very good. However, there are some deviations in the low- and the medium-density regions by at most $20 \%$ if we scale the longitudinal axes so as to adjust the values in the high-density region.

In $\mathrm{C}_{2} \mathrm{H}_{6}$, the vibrational energy relaxation rate in the $S_{2}$ state at $385 \mathrm{~K}$ shows the same density dependence of those in the ground state while the rate in the $S_{2}$ state become 

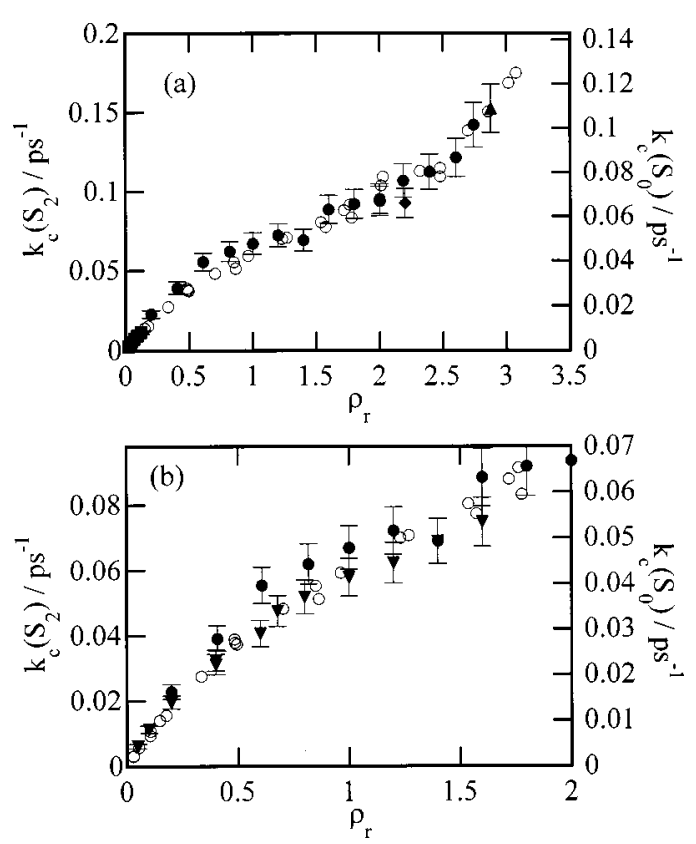

FIG. 6. Solvent density dependence of the vibrational energy relaxation rates in $\mathrm{C}_{2} \mathrm{H}_{6}$. Scaling factor is 1.4. (a) The results at the lower temperatures. Filled marks: $S_{2}$ state. Open circles: $S_{0}$ state in the literature (Ref. 15). $\mathbf{\square}$ : $342 \mathrm{~K} ; \bullet: 323 \mathrm{~K} ; \quad: 308 \mathrm{~K} ; \boldsymbol{\Delta}: 298 \mathrm{~K}$. (b) Temperature effect. $\bigcirc: k_{c}\left(S_{0}\right)$ in the literature (Ref. 15). $-k_{c}\left(S_{2}\right)$ at $323 \mathrm{~K} . \boldsymbol{\nabla}: k_{c}\left(S_{2}\right)$ at $385 \mathrm{~K}$.

faster at $323 \mathrm{~K}$ in the low- and the medium-density region. The temperature effect appears smaller in the case of $\mathrm{CO}_{2}$ and $\mathrm{Xe}$, and in both solvents, the rates in the $S_{2}$ state in the medium-density region are larger than those in the ground state at the same density, if we scale the rates in two electronic states as is explained above.
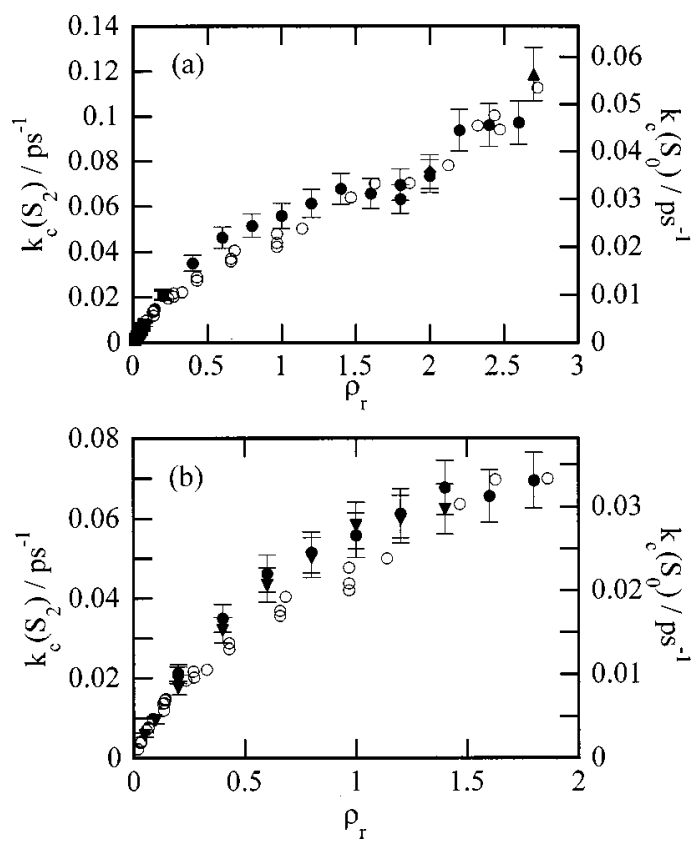

FIG. 7. Solvent density dependence of the vibrational energy relaxation rates in $\mathrm{CO}_{2}$. Scaling factor is 2.1. (a) The results at the lower temperatures. (b) Temperature effect. All marks are the same as those in Fig. 6.

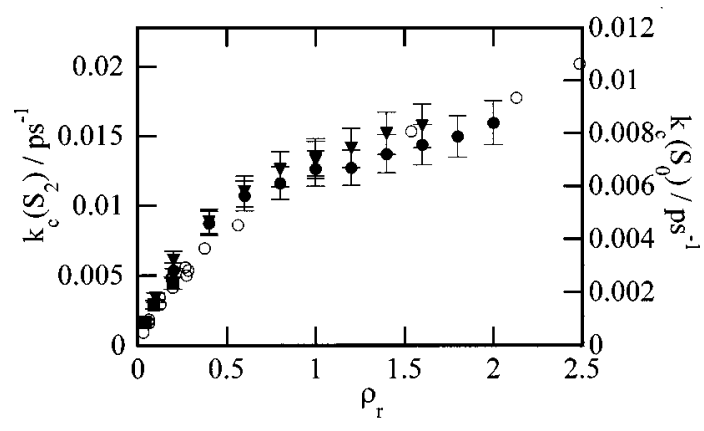

FIG. 8. Solvent density dependence of the vibrational energy relaxation rates in Xe. Scaling factor is 1.9. All marks are the same as those in Fig. 6.

\section{DISCUSSIONS}

\section{A. The interpretation of the solvent density dependence}

The most important finding of our results on the vibrational energy relaxation rates of azulene in three supercritical fluids is that the solvent density dependence is similar for both electronic states, accepting the differences of about $20 \%$. This indicates that the density dependence of the relaxation rates in both states is dominated nearly by the same factor that is the collision frequency in the context of the IBC model [Eq. (1)]. The binary and the correlated multiple collisions have different power spectra in general, and the power spectrum of the latter itself changes with solvent density. ${ }^{33}$ The relative probability of the binary and the correlated collision also depends on solvent density. ${ }^{34}$ Therefore, the contribution of correlated multiple collisions is likely to cause the different density dependence of the relaxation rates of the $S_{0}$ and the $S_{2}$ states, if the difference of the absolute rates of both states are due to the change of the intramolecular vibrational frequency as is proposed in paper I. As was previously described, the solvent density effects on the absorption and the fluorescence spectra of the $S_{0}-S_{2}$ transition are small, which indicates that the solute-solvent interaction is insensitive to the electronic states. As a result, the solute-solvent collision frequencies in both states follow the similar density dependence, and the density dependence of the relaxation rates will be similar in the IBC model. Therefore, our experimental results can be explained by the $I B C$ model for the vibrational energy relaxation in fluids, although our results do not exclude all other possibilities. The difference of the absolute relaxation rates between the two electronic states can be understood as the dependence of the vibrational quenching efficiency in the gas phase on the electronic states of the solute. Although a small difference $(\sim 20 \%)$ of the density dependence is found in the low- and the medium-density regions experimentally, we consider that it should be considered as perturbations to the IBC model.

Before discussing the small difference, it would be helpful to explain the inversely $s$-shaped density dependence of the relaxation rates. There have been a tremendous number of studies on the inversely $s$-shaped density dependence in supercritical fluids. ${ }^{35,36}$ Although most of these studies focus on the solvatochromism or the thermodynamic properties, 
the inversely $s$-shaped density dependence was already found in many studies even in the field of vibrational energy relaxation. For example, Adelman et al. ${ }^{9}$ Schwarzer et al. ${ }^{16}$ Goodyear and Tucker, ${ }^{21}$ and Egorov and Skinner ${ }^{22}$ have found it numerically, and Schwarzer et al., ${ }^{15}$ and Uhdahl et al. ${ }^{19}$ observed it experimentally. In many studies, the global behavior of the density dependence of the relaxation rates is understood in terms of the local density enhancement as is described below. ${ }^{16,21,35}$ The structure of dense fluids, including ordinary organic solvents in the ambient condition, is mainly determined by the repulsive part of the intermolecular potential. ${ }^{33,34}$ On the other hand, the attractive part of the intermolecular potential plays a significant role in the structure of the low- and medium-density fluids. ${ }^{16,35-37}$ For example, the strong attractive potential between the solute and the solvent can enhance the local density around the solute, which is often called clustering. This difference of the determining factor of the equilibrium structure is essential to the understandings of the solvation in low- and medium-density fluids.

When there are no attractive interactions between molecules, for example, in the case of hard sphere fluids, the collision frequency shows a positive curvature at every density, and the density dependence rapidly increases in the high-density region. ${ }^{34}$ Suppose that the attractive potential between the solute and the solvent is added as a perturbation. ${ }^{38}$ Since the structure of the fluid is determined by the packing efficiency in the high-density region, the attractive potential between the solute and the solvent affects little the collision frequency. On the other hand, the attractive interaction between the solute and the solvent can gather the solvent molecules around the solute when the packing of the solvents is loose, i.e., in the low- and the medium-density regions. As a result, the collision frequency is enhanced in the low- and the medium-density regions, which results in the inversely $s$-shaped density dependence of the collision frequency.

The different temperature effect on the vibrational energy relaxation rates among three fluids is somewhat confusing in relation to the above-mentioned local density effect. When the temperature rises, the initial slope of the density dependence of the relaxation rates in the $S_{2}$ states become smaller in $\mathrm{C}_{2} \mathrm{H}_{6}$. Since the local density enhancement is caused by the attractive interaction between the solute and the solvent, its effect should be reduced at higher temperatures. Therefore, we consider the temperature effect observed in $\mathrm{C}_{2} \mathrm{H}_{6}$ reasonable. In fact, Schwarzer et al. found a large temperature effect on the density dependence of the relaxation rate, and they succeeded in explaining it by the IBC model. ${ }^{16 a}$ On the other hand, the temperature effect in $\mathrm{CO}_{2}$ and $\mathrm{Xe}$ is different from that in $\mathrm{C}_{2} \mathrm{H}_{6}$. At present, we have no explanation for the different temperature effect. The temperature dependence of the vibrational quenching efficiency may affect the different temperature effects. Considering our experimental error, the improvement of the experimental method will be required to discuss the small temperature effect in detail. In addition, it is desirable to construct a calibration curve taking the temperature dependence of the relaxed fluorescence band shape into account. Anyway, it seems better to seek other reasons for the small difference in the density dependence of the relaxation rates in the $S_{0}$ and the $S_{2}$ states.

Besides the temperature effect, we consider three factors that may cause the different density dependence of the relaxation rates in the $S_{0}$ and the $S_{2}$ states. The first one is the difference of the collision frequencies between two electronic states. The second one is the change of the effective channel as proposed by Heidelbach et al. ${ }^{17}$ The last one is the effect of the heat diffusion among the solvents. ${ }^{39-41}$

We can qualitatively explain the difference of the density dependence of the relaxation rates in the $S_{0}$ and the $S_{2}$ states by assuming that the solute-solvent attractive interaction is somewhat stronger when the solute is in the $S_{2}$ state. Since a stronger attractive interaction causes a larger clustering effect, the collision frequency and the relaxation rate in the $S_{2}$ state become larger in the low- and the mediumdensity regions. On the other hand, the collision frequency is little affected by the attractive interaction in the high-density region, which results in the similar density dependence of the relaxation rates. The problem is how large the attractive potential is in the excited state. Since the $S_{0}$ and the $S_{2}$ states are combined by the direct photoinduced transitions, we can estimate the difference of the solute-solvent interactions of $S_{0}$ and $S_{2}$ states from the frequency shift of the optical spectra. According to the study on the fluorescence excitation spectra of the azulene- $\mathrm{C}_{2} \mathrm{H}_{6}$ and the azulene-Xe van der Waals (vdW) $1: 1$ complexes, the shifts of the $0-0$ bands are $-45.5 \mathrm{~cm}^{-1}$ and $-54 \mathrm{~cm}^{-1}$ for azulene- $\mathrm{Xe}^{42}$ and azulene- $\mathrm{C}_{2} \mathrm{H}_{6}{ }^{43}$ complexes, respectively. These shifts correspond to the differences of the binding energies of the solute-solvent binary potentials, if the bottom of the solutesolvent potential of the excited state exists within the Franck-Condon accessible region. If we estimate the effect of this change of the attractive potential from the Boltzmann factor at $385 \mathrm{~K}$, neglecting the correlation between solvent molecules, the local density enhancement in the excited state is expected to be $18-22 \%$ larger than that in the ground state. These values happen to agree with the difference between the density dependence of the relaxation rates in the $S_{0}$ and the $S_{2}$ states. However, the fluorescence shifts in fluids are no larger than $+5 \mathrm{~nm}\left(\sim-400 \mathrm{~cm}^{-1}\right)$ at the highest density of $\mathrm{C}_{2} \mathrm{H}_{6}$ in our experiment. If we divide the redshift equally to the solvents in the first solvation shell, the amount of the redshift per one solvent molecule is smaller than $50 \mathrm{~cm}^{-1}$ (the coordination number is about $12 \mathrm{~mol}-$ ecules when the solute and the solvent is identical. Provided the difference of the sizes, the coordination number will be larger than 12). This difference may indicate the importance of the anisotropy of the interaction potential.

Heidelbach et al. performed computer simulations on the vibrational energy relaxation of azulene in the $S_{0}$ state, and found that several low-frequency modes exclusively act as the relaxation channel in the low-density region, whereas higher-frequency modes can contribute to the vibrational energy relaxation in the high-density region to some degree. This may also be the reason for the difference of the density dependence of the relaxation rates in the $S_{0}$ and the $S_{2}$ states. We proposed in paper I that the decrease of the intramolecu- 
lar vibrational frequency can be a reason for the fast vibrational energy relaxation in the $S_{2}$ state. ${ }^{24}$ However, the effect of the existence of the low-frequency modes would decrease in the high-density region if the effective channel of the energy relaxation shifts to the higher-frequency modes. As a result, the density dependence of the vibrational energy relaxation rates in the $S_{2}$ state might be smaller than that in the $S_{0}$ state at $\rho_{r}=1.0-2.0$.

The heat flow among the solvent molecules is another interesting problem. Since energy is a conserved variable, it can relax only by the diffusion among the solvents. Therefore, the transient temperature can be higher than the bulk one when the heat diffusion is slow, which can produce an additional slow component of the energy relaxation. From the time profile of the energy relaxation and the molecular dynamics simulation, Schwarzer et al. concluded that the solvent-solvent energy transfer does not affect the observed energy relaxation in the case of azulene in the $S_{0}$ state. $^{15,17}$ Since the absolute relaxation rate in the $S_{2}$ state is larger than that in the $S_{0}$ state, there is no strong evidence of the observation of the heat flow in our experiment. However, there may be some influence of the heat flow among the solvent on the relaxation rates in the $S_{2}$ state, since the solute-solvent energy transfer is faster.

Anyway, the most important result of this work is that the density dependence of the vibrational energy relaxation rates of azulene in the $S_{0}$ and the $S_{2}$ states are similar, which is consistent with the prediction of the IBC model.

\section{B. The implication of the isolated binary collision model for the vibrational energy relaxation in fluids}

We have shown so far that the density dependence of the vibrational energy relaxation of azulene is explained in the IBC model approximately. In the following part of this paper, we shall discuss the physical background that may exist behind the IBC model. Parts of the discussion below were already presented in our paper of the molecular dynamics simulation on the solute diffusion ${ }^{44}$ and the nonpolar solvation dynamics. $^{23}$

The population relaxation time $\left(T_{1}\right)$ of an oscillator is described by the following equation (called Landau-Teller theory) in the weak coupling limit:

$$
T_{1}^{-1} \propto \operatorname{Re} \int_{0}^{\infty} d t e^{i \omega t}\langle F(0) F(t)\rangle .
$$

In Eq. (3), $\omega$ is the frequency of the oscillator, and $F(t)$ is the random force from the solvent along the vibrational normal coordinate. Equation (3) states that the vibrational population relaxation occurs through the resonance with the power spectrum of the random force from the solvent. In the high-density region, the whole functional form of $\langle F(0) F(t)\rangle$ is not determined by the binary collision between solute and solvent. For example, the IBC model (Enskog theory) does not explain the self-diffusion coefficient, which is inversely proportional to the zero-frequency part of the power spectrum of the random force. ${ }^{34}$ However, only the quickly varying short time part of $\langle F(0) F(t)\rangle$ is effec- tive to the vibrational energy relaxation, since the frequency of the intramolecular vibration is higher than that of the thermal motion of the solvent in general.

There have been some computer simulations which shows that the quickly varying short time behavior of $\langle F(0) F(t)\rangle$ is dominated by the binary interaction. ${ }^{7,10,11,12,44}$ Based on Eq. (3), Chesnoy and Weis performed a molecular dynamics simulation to obtain $\langle F(0) F(t)\rangle$. They found that the three-body interference does not affect the highfrequency part of the power spectrum and the density dependence of the relaxation rate correlates with the radial distribution function at an adequate distance. ${ }^{7}$ Adelman et al. also showed that the IBC-like picture emerges when the initial part of the time correlation function of the random force $(\langle F(0) F(t)\rangle)$ does not depend on the solvent density. ${ }^{9}$ If the functional form of the initial part does not depend on the solvent density, the relaxation rate is factorized into the static contribution $\left(\left\langle|F(0)|^{2}\right\rangle\right)$ and the density independent dynamic contribution. Adelman et al. proposed that the IBClike picture is reproduced by interpreting $\left\langle|F(0)|^{2}\right\rangle$ as the collision frequency. Although the density independence of the initial part itself depends on the intermolecular potentials, we have shown by molecular dynamics simulations that the functional form of the initial part of the time correlation function of the random force on the whole molecule does not depend on the solvent density in the case of Lennard-Jones 12-6 potential. $^{44}$

The binary character of the short time part of $\langle F(0) F(t)\rangle$ is often ascribed to the fact that it is mainly determined by the repulsive interaction. There are two reasons for the dominance of the repulsive interaction. Since the force is the derivative of the interaction potential, the large slope in the repulsive region leads to a large contribution to the force. In addition, quickly varying character of the repulsive interaction causes fast relaxation of $\langle F(0) F(t)\rangle$. The situation may be different in the presence of strong attractive interactions such as hydrogen bonding. Then, why the short time behavior of the fluctuation of the repulsive force has a binary character? In addition, it seems that there exists a small gap between $Z$ and $\left\langle|F(0)|^{2}\right\rangle$, because the former is the two-body quantity, whereas the latter is the three-body quantity in principle.

The vibrational energy relaxation is sometimes discussed in parallel with the nonpolar solvation dynamics, since $\langle F(0) F(t)\rangle$ can be treated in a similar way as the solvation correlation function of the nonpolar solvation dynamics. Recently we performed an extensive molecular dynamics simulation on the nonpolar solvation dynamics in Lennard-Jones fluids. ${ }^{23}$ We have explicitly demonstrated that the time constant of the initial part of the solvation correlation function is determined by the curvature of the free energy surface, which can be estimated from the ratio of the solvent induced static shift to the static fluctuation. In particular, when the repulsive part of the potential changes on excitation, the static fluctuation is approximately proportional to the static shift, and the functional form of the initial part of the solvation correlation function does not depend on the solvent density. In other words, the dynamic correlation between solvent molecules does not exist in the initial part of the solvation 
correlation function since the static correlation between solvent molecules is absent in the static fluctuation. If this idea is applicable to the vibrational energy relaxation, the absence of the static correlation between solvent molecules in $\left\langle|F(0)|^{2}\right\rangle$ accompanies the density independence of the functional form of the initial decay of $\langle F(0) F(t)\rangle$. In such a case, $\left\langle|F(0)|^{2}\right\rangle$ can be interpreted as the collision frequency, since it becomes a two-body quantity effectively. This behavior of the initial part of $\langle F(0) F(t)\rangle$ corresponds to the large-wave-number limit of the static and the dynamic structure factor of simple fluids, ${ }^{34}$ and the steepness of the repulsive potential plays a key role in the validity of the IBC model. It is to be noted that our discussion on the relationship between the static fluctuation and the initial part of its relaxation in the case of nonpolar solvation dynamics applies only when the contributions of the attractive and the repulsive interactions are properly divided. ${ }^{23}$ In the presence of both contributions, such as the Lennard-Jones interaction, the static fluctuation may not be binary due to the contribution of the attractive part, while the time correlation function can possess the binary part owing to the repulsive interaction.

We shall comment here against the prevailing idea that the IBC model must break down automatically when the collision frequency between the solute and the solvent approaches the intramolecular vibrational frequency. The force from the solvent molecules along the vibrational coordinate is the sum of the contributions of each solvent as follows:

$$
F(t)=\sum_{i} f_{i}(t)
$$

where $f_{i}$ is the force from the $i$ th solvent molecule. The time correlation function of $F(t)$ can be divided into the self- and the cross-parts as follows:

$$
\langle F(0) F(t)\rangle=\sum_{i}\left\langle f_{i}(0) f_{i}(t)\right\rangle+\sum_{i \neq j}\left\langle f_{i}(0) f_{j}(t)\right\rangle .
$$

The cross term (the second term of rhs) vanishes in the absence of the correlation between collisions. In such a case, $\langle F(0) F(t)\rangle$ and $T_{1}^{-1}$ are described as the sum of the contribution of each solvent molecule. The assumption that the collision frequency is smaller than the vibrational frequency is not used in the above discussion. However, there have been some studies that state that the IBC model must break down when the collision frequency between the solute and the solvent approaches the intramolecular vibrational frequency.

Zwanzig calculated the effect of the interference between two collisions based on Eq. (3), and proposed that the interference effect is important when the collision frequency approaches the intramolecular vibrational frequency. ${ }^{3}$ In his calculation, he assumed a repulsive correlation between two collisions, that is, only one solvent molecule can collide with the solute molecule at a time [see Eq. (26) of Ref. 3]. This assumption is essential to his calculation, and there is no interference effect in the absence of repulsive correlation between solvent molecules. Therefore, his calculation does not contradict with our idea that the absence of the correlation between the solvent molecule in the static fluctuation of the random force is essential to the IBC model. The important point is whether there is a repulsive correlation between two collisions. We consider that the repulsive correlation in this model will be relaxed in the real systems, since there are many collision sites in a solute molecule, which is partially proved by our simulation previously mentioned. ${ }^{23}$

Shin and Keizer calculated the interference effect between two adjacent collisions, and showed that there is a significant interference effect even in the absence of the correlation between collisions. ${ }^{4}$ We consider that it is because they calculated the interference effect between two adjacent collisions. There is no interference effect between two arbitrary collisions when there is no correlation between the collisions. On the other hand, there is a time correlation between two adjacent collisions simply because they are adjacent. The time scale of the correlation is on the order of $Z^{-1}$, and contributes to the power spectrum at the frequency $Z$. In our opinion, it is their choice of the pair of collisions that produced the interference effect between them.

Our discussion in this subsection is based on Eq. (3) so far. However, there are some relaxation mechanisms beyond Eq. (3), which may lead to the breakdown of the IBC model. The first one is the $V-V$ transfer. It is shown experimentally and theoretically that the IBC model breaks down in the case of $V-V$ transfer of small $\left(<150 \mathrm{~cm}^{-1}\right)$ frequency mismatch. $^{45,46}$ The second one is the solvent assisted IVR (intramolecular energy redistribution). Heidelbach et al. found that the solvent assisted IVR is the dominant mechanism in their molecular dynamics simulation on the vibrational energy relaxation of azulene in the $S_{0}$ state in fluids, and that the effective channel depends on the solvent density. ${ }^{17}$ In addition, the higher order perturbation than Eq. (3) is recently claimed to be important. ${ }^{47,48}$ The roles of the above effects in the vibrational energy relaxation of azulene in the $S_{2}$ state are not clear at present, and further studies will be required.

\section{SUMMARY}

We have determined the vibrational energy relaxation rates of $\mathrm{S}_{2}$ azulene in $\mathrm{C}_{2} \mathrm{H}_{6}, \mathrm{CO}_{2}$, and $\mathrm{Xe}$, from the gaslike to the liquidlike densities by the time-resolved fluorescence spectroscopy. Although the absolute rates in the $S_{2}$ state are 1-2 times larger than those in the $S_{0}$ state, the density dependence in the $S_{2}$ state is approximately similar to that in the $S_{0}$ state. It can be understood by the IBC model for the vibrational energy relaxation, that is, the density dependence of the relaxation rates are determined by that of the collision frequency, although there remain some other possibilities for the explanation. The ratio of the relaxation rate in the $S_{2}$ state to that in the $S_{0}$ states is about $20 \%$ larger in the lowand medium-density regions, compared with that in the highdensity region. Possible reasons for this small discrepancy of the density dependence were presented. However, further studies including the detailed measurement of the temperature effect, the examination of the precise interaction potential and the determination of the detailed time profile will be required for discussion of the small difference of the density dependence quantitatively. We also discussed the physical backgrounds for the IBC model for the vibrational energy relaxation in fluids based on the Landau-Teller theory and 
molecular dynamics simulations. We proposed that the absence of the static correlation between collisions is essential to the validity of the IBC model.

\section{ACKNOWLEDGMENTS}

We are grateful to Dr. Y. Yoshimura (Kyoto University) for the use of the UV absorption spectrometer and the highpressure optical cells. T.Y. gratefully acknowledges a research fellowship from the Japan Society for the Promotion of Science (JSPS) for Young Scientists. This work is supported by CREST (Core Research for Evolutional Science and Technology) of Japan Science and Technology (JST) and by the Research Grant-in-Aid from the Ministry of Education, Science, and Culture (No. 11640504).

${ }^{1}$ C. Kittel, J. Chem. Phys. 14, 614 (1946).

${ }^{2}$ T. A. Litovitz, J. Chem. Phys. 26, 469 (1957); W. M. Madigosky and T. A. Litovitz, ibid. 34, 489 (1961).

${ }^{3}$ R. Zwanzig, J. Chem. Phys. 34, 1931 (1961).

${ }^{4}$ H. K. Shin and J. Keizer, Chem. Phys. Lett. 27, 611 (1974).

${ }^{5}$ D. W. Oxtoby, Annu. Rev. Phys. Chem. 32, 77 (1981); D. W. Oxtoby, Adv. Chem. Phys. 47, 487 (1981).

${ }^{6}$ M. Châtelet, J. Kieffer, and B. Oksengorn, Chem. Phys. 79, 413 (1983); B. Oksengorn, ibid. 140, 233 (1990).

${ }^{7}$ J. Chesnoy and J. J. Weis, J. Chem. Phys. 84, 5378 (1986).

${ }^{8}$ J. Chesnoy and G. M. Gale, Adv. Chem. Phys. 70, 295 (1988), and references therein.

${ }^{9}$ S. A. Adelman, R. Muralidhar, and R. H. Stote, J. Chem. Phys. 95, 2738 (1991); S. A. Adelman, R. H. Stote, and R. Muralidhar, ibid. 99, 1320 (1993).

${ }^{10}$ G. Goodyear and R. M. Stratt, J. Chem. Phys. 107, 3098 (1997).

${ }^{11}$ B. M. Ladanyi and R. M. Stratt, J. Phys. Chem. A 102, 1068 (1998).

${ }^{12}$ R. E. Larsen and R. M. Stratt, J. Chem. Phys. 110, 1036 (1999).

${ }^{13}$ M. E. Paige and C. B. Harris, Chem. Phys. 149, 37 (1990).

${ }^{14}$ K. E. Schultz, D. J. Russell, and C. B. Harris, J. Chem. Phys. 97, 5431 (1992).

${ }^{15}$ D. Schwarzer, J. Troe, M. Votsmeier, and M. Zerezke, J. Chem. Phys. 105, 3121 (1996); Ber. Bunsenges. Phys. Chem. 101, 595 (1997).

${ }^{16}$ D. Schwarzer, J. Troe, and M. Zerezke, J. Chem. Phys. 107, 8380 (1997); J. Phys. Chem. A 102, 4207 (1998).

${ }^{17}$ C. Heidelbach, I. I. Fedchenia, D. Schwarzer, and J. Schroedar, J. Chem. Phys. 108, 10152 (1998); C. Heidelbach, J. Schroedar, D. Schwarzer, and V. S. Vikhrenko, Chem. Phys. Lett. 291, 333 (1998); C. Heidelbach, V. S. Vikhrenko, D. Schwarzer, and J. Schroedar, J. Appl. Phys. 110, 5286 (1999); C. Heidelbach et al., ibid. 111, 8022 (1999).

${ }^{18}$ J. Benzler, S. Linkersdörfer, and K. Luther, J. Chem. Phys. 106, 4992 (1997).

${ }^{19}$ R. S. Uhdahl et al., J. Chem. Phys. 107, 3747 (1997); D. J. Myers, R. S. Urdahl, B. J. Cherayil, and M. D. Fayer, ibid. 107, 9741 (1997); D. J. Myers et al., ibid. 109, 5971 (1998).

${ }^{20}$ B. J. Cherayil and M. D. Fayer, J. Chem. Phys. 107, 7642 (1997).

${ }^{21}$ G. Goodyear and S. C. Tucker, J. Chem. Phys. 110, 3643 (1999).

${ }^{22}$ S. A. Egorov and J. L. Skinner, J. Chem. Phys. 112, 275 (2000).
${ }^{23}$ T. Yamaguchi, Y. Kimura, and N. Hirota, J. Chem. Phys. 111, 4169 (1999).

${ }^{24}$ T. Yamaguchi, Y. Kimura, and N. Hirota, J. Chem. Phys. (in press).

${ }^{25}$ Y. Kimura, T. Yamaguchi, and N. Hirota, Chem. Phys. Lett. 303, 223 (1999).

${ }^{26}$ Y. Kimura and N. Hirota, J. Chem. Phys. 111, 5474 (1999).

${ }^{27}$ T. Yamaguchi, Y. Kimura, and N. Hirota, J. Phys. Chem. A 101, 9050 (1997).

${ }^{28}$ H. Ooe, Y. Kimura, M. Terazima, and N. Hirota, J. Phys. Chem. A 103, 7730 (1999).

${ }^{29}$ In some experiments we used the cell of the same design made of SUS 304.

${ }^{30}$ Xe: V. A. Rabinovich, A. A. Vasserman, V. I. Nedstup, and L. S. Veksler, Thermophysical Properties of Neon, Argon, Krypton, and Xenon (Hemisphere, Washington, DC, 1988); $\mathrm{C}_{2} \mathrm{H}_{6}$ below $50 \mathrm{MPa}$ : B. A. Younglove and J. F. Ely, J. Phys. Chem. Ref. Data 16, 577 (1987); $\mathrm{C}_{2} \mathrm{H}_{6}$ above 50 MPa: Von M. Rätzsch and R. Findeisen, Z. Phys. Chem. (Leipzig) 261, 935 (1980); $\mathrm{CO}_{2}$ : F. H. Huang, M. H. Li, K. E. Starling, and F. T. H. Chun, J. Chem. Eng. Jpn. 18, 490 (1985).

${ }^{31}$ L. Reynolds et al., J. Phys. Chem. 100, 10037 (1996).

${ }^{32}$ The value of $600 \mathrm{~cm}^{-1}$ appears large for the difference of the intramolecular thermal energy. Since the intramolecular energy dependence of the fluorescence band shape is small at smaller $\langle E(t)\rangle$, the large error is expected for $600 \mathrm{~cm}^{-1}$. However, the estimation of $E_{b}$ will affect little the relaxation rate since the rate changes only less than $10 \%$ if we interpret the change of the relaxed fluorescence as the solvent induced broadening.

${ }^{33}$ J. W. Weeks, D. Chandler, and H. C. Andersen, J. Chem. Phys. 54, 5237 (1971).

${ }^{34}$ J. P. Hansen and I. R. McDonald, Theory of Simple Liquids, 2nd ed. (Academic, London, 1986), and references therein.

${ }^{35}$ O. Kajimoto, Chem. Rev. 99, 355 (1999), and references therein.

${ }^{36}$ S. C. Tucker, Chem. Rev. 99, 391 (1999), and references therein.

${ }^{37}$ Y. Kimura and Y. Yoshimura, Mol. Phys. 72, 279 (1991).

${ }^{38}$ Although the attractive interaction between solvents enhances the clustering effect (Refs. 23 and 27), the local density enhancement itself can arise solely from the attractive interaction between the solute and the solvent. In fact, the local density enhancement in supercritical fluids is often analyzed by the Langmuir adsorption model (Ref. 35), which totally neglect the attractive correlation between solvent molecules. Further studies will be required for the quantitative discussion on the role of the attractive potential between solvents in the clustering effect.

${ }^{39}$ U. Sukowski, A. Seilmeier, T. Elsaesser, and S. F. Fisher, J. Chem. Phys. 93, 4094 (1990); T. Elsaesser and W. Kaiser, Annu. Rev. Phys. Chem. 42, 83 (1991), and references therein.

${ }^{40}$ K. Iwata and H. Hamaguchi, J. Phys. Chem. 101, 632 (1997).

${ }^{41}$ T. Okazaki, N. Hirota, and M. Terazima, J. Chem. Phys. 110, 11399 ().

${ }^{42}$ O. K. Abou-Zied, H. K. Sinha, and R. P. Steer, J. Phys. Chem. 100, 4375 (1996).

${ }^{43}$ H. K. Sinha et al., Chem. Phys. Lett. 230, 547 (1994).

${ }^{44}$ T. Yamaguchi, Y. Kimura, and N. Hirota, Mol. Phys. 94, 527 (1998).

${ }^{45}$ J. J. Andrew et al., Chem. Phys. 139, 369 (1989).

${ }^{46}$ G. T. Evans and P. A. Madden, Mol. Phys. 74, 1171 (1991).

${ }^{47}$ M. Shiga and S. Okazaki, J. Chem. Phys. 109, 5511 (1998); ibid. 111, 5390 (1999).

${ }^{48}$ B. J. Cherayil, J. Chem. Phys. 111, 6471 (1999). 